\title{
Management of Atrial Fibrillation after Cardiac Surgery
}

Sean M Conte, BA MBBS [1], Daniel S Florisson, BS MBBS [2], Joshua A De Bono, BS MBBS [2], Reece A Davies, BS MBBS [3], Andrew E Newcomb, MBBS FRACS [2]

\section{Affiliations:}

[1] Department of Medicine, St Vincent's Hospital Sydney, 390 Victoria St, Darlinghurst, NSW 2010, Australia

[2] Department of Cardiothoracics, St Vincent's Hospital Melbourne, 41 Victoria Pde, Fitzroy, VIC 3141, Australia

[3] Department of Cardiothoracics, John Hunter Hospital, Lookout Rd, New Lambton Heights, NSW 2305, Australia

\section{Corresponding Author:}

Sean M Conte

1 Turner St, Redfern, NSW 2016, Australia

$+61.423 .037 .106$

sean.m.conte@gmail.com

Conflict of interest: none declared

Sources of funding: no funding provided

\section{SUMMARY}

A best evidence topic in cardiac surgery was written according to a structured protocol addressing the question "for post-cardiac surgery atrial fibrillation, do clinical outcomes differ between rate or rhythm control strategies?" Altogether, 2174 papers were found using the reported searches, of which five represented the best evidence to answer the clinical question. Hospital length of stay ranged from 5.0-13.2 days for rate control and 5.2-10.3 days for rhythm control. Freedom from AF at follow-up was achieved in $84.2-91 \%$ and $84.2-96 \%$ in rate and rhythm control groups respectively. Minimal serious adverse events were noted in all studies analysed and there was no difference between rate and rhythm control

This is the author manuscript accepted for publication and has undergone full peer review but has not been through the copyediting, typesetting, pagination and proofreading process, which may lead to differences between this version and the Version of Record. Please cite this article as doi: 10.1111/imj.14281

This article is protected by copyright. All rights reserved. 
groups. We conclude that in the management of post-cardiac surgery atrial fibrillation, rate control and rhythm control are equivalent in terms of hospital length of stay, freedom from arrhythmia at follow-up, and complication rates.

\section{KEY WORDS}

review; atrial fibrillation; rate control; rhythm control; cardiac surgery; post-operative atrial fibrillation; post-operative complication

\section{MANUSCRIPT}

\section{Introduction}

In patients with atrial fibrillation (AF) following cardiac surgery is rate control or rhythm control superior in terms of clinical outcomes such as hospital length of stay, rates of recurrence, freedom from arrhythmia at follow-up, and rates of complications? A best evidence topic was constructed according to a structured protocol.[1]

\section{Search strategy \& outcome}

A total of 604 papers were found searching Medline and Embase (498 and 106 respectively) using Ovid from 1990 to August 2018 and 1570 were found searching PubMed. The search parameters were \{[Atrial Fibrillation/ or Atrial Flutter/] and [Cardiac Surgery.mp or Thoracic procedures/] and [Postoperative complications/ $]\}$ on Medline, $\{[($ atrial fibrillation/ OR atrial flutter/) $]$ and [antiarrhythmic agent/] on Embase $[($ Cardiac surgery OR Heart Surgery) $]\}$ and $\{[$ Heart Surgery/] and [(atrial fibrillation OR atrial flutter)] and [antiarrhythmic agent]\} on PubMed. From these, five papers were identified that provided the best evidence to answer the question. Four of the papers were randomised trials and one was a retrospective cohort study. The trials were evaluated for quality using the widely-validated Jadad criteria and Newcastle-Ottawa scale for randomised and non-randomised studies respectively.[2,3] 


\section{Results}

Gillinov, et al[4] conducted a multicentre randomised trial on this topic in which they evaluated rate control alone with beta-blockers or calcium-channel blockers versus rhythm control with amiodarone in 523 patients with new AF following cardiac surgery. The authors found no significant difference in terms of total days in hospital within 60 days of randomisation ( 5.1 v 5.0 days, $p=0.76$ ), hospital discharge readiness from an atrial fibrillation perspective ( 4.0 days for both groups, $\mathrm{p}=0.99$ ), readmission rates ( $18.5 \%$ for both groups, $\mathrm{p}=0.99)$, and rates of serious adverse events or death $(24.8 \mathrm{v} 26.4$ per 100 patient months, $\mathrm{p}=0.61$ ). They found that the rhythm control group was slightly more likely to have been free from $\mathrm{AF}$ at 60 days for the previous 30 days $(93.8 \% \mathrm{v} 97.9 \%, \mathrm{p}=0.02)$. The trial had high rates of noncompliance/crossover between rate and rhythm control groups $(26.7 \%$ and $24 \%$ with $80 \%$ and $48 \%$ of these changing therapies in the index hospitalisation, respectively) mostly due to drug ineffectiveness in the rate-control group and drug side effects in the rhythm-control group. This hampers the trial's validity but emphasises the complexity of post-operative AF management. The trial was randomised in a sequential 1:1 fashion using a Electronic Data Capture (EDC) system after a checklist of inclusion and exclusion criteria were completed and verified. This randomisation method was rigorous and carried out by a dedicated research staff member across all sites. Treatment was unblinded due to practical limitations. There were 19 patients that withdrew consent following randomisation however they were included in the intention-to-treat analysis. The paper scores a three on the Jadad criteria and is therefore the largest and highest-quality study included in this review.

Lee, et al[5] randomised 50 patients with new-onset AF following cardiac surgery to receive either rate or rhythm control and found no significant difference between strategies in terms of time to conversion to sinus rhythm $(11.8 \pm 3.9 \mathrm{v} 11.2 \pm 3.2$ hours, $\mathrm{p}=0.8)$, maintenance of sinus rhythm at 2-month follow-up (91\% v 96\%), and recurrence of AF in hospital $(57 \% \mathrm{v} 30 \%, \mathrm{p}=0.24)$. They found a significant reduction in hospital length of stay for patients in the rhythm control arm (13.2 $\pm 2.0 \mathrm{v} 9.0 \pm 0.7$ days, $\mathrm{p}=0.05) .12$ of the 27 patients in the rhythm-control group received propafenone, an agent that promotes 1:1 atrioventricular node conduction, and thus were also commenced on rate control. Rate control was allowed in the rhythm-control group if heart rate exceeded 110 beats per minute. Finally, five of the patients in the rate-control arm crossed over to rhythm-control due to relapsing AF (three), contraindication to anticoagulation (one), and failure of rate-control (one). Again the trial was randomised 
but unblinded due to logistical reasons and there is no description of the randomisation method. There were no withdrawals or dropouts and all randomised patients were included in the analysis garnering a Jadad score of two.

Cochrane, et al compared digoxin to amiodarone for new-onset post-cardiac surgery atrial fibrillation or flutter in a randomised trial of 30 patients.[6] They found no significant difference in terms of how many patients remained in AF 24 hour after initiation of therapy $(20 \% \mathrm{v} 6.67 \%, 3 \mathrm{v} 1$ patient, $\mathrm{p}>0.05)$ and noted that both agents were effective in reducing heart rate (144 to $95 \mathrm{v} 146$ to $89, \mathrm{p}>0.05$ ), especially within the first 6 hours of therapy, and detected no major adverse reactions in either group. Digoxin is no longer standard of care rate-control and this trial only evaluated short-term (24-hour) outcomes but suggests both methods were equiefficacious in the initial management of post-cardiac surgery AF. The patients were randomised "on the basis of their hospital record number" and the trial was unblinded due to logistics. No patients withdrew and there was only one crossover (one patient in the rate-control group who failed to revert to sinus rhythm), thus the trial scores a two according to the Jadad criteria.

Hjelms compared procainamide to digoxin in terms of time to conversion of new post-cardiac surgery atrial fibrillation in a randomised trial of 30 patients and found significantly reduced time to conversion with procainamide versus digoxin ( $40 \mathrm{v} 540$ minutes, $\mathrm{p}<0.002)$.[7] They also noted higher rate of successful conversion to sinus rhythm within 12 hours of treatment in the procainamide group $(60 \% \mathrm{~V}$ $93 \%, \mathrm{p}<0.05)$. No difference in recurrence of AF after conversion was found $(22.2 \% \mathrm{v} 36 \%$, not significant). There were no appreciable differences in rates of serious complications. The use of digoxin for rate-control is outdated thus limiting the generalisability of these results to modern practice and, like Cochrane's study, this trial evaluated only very short term outcomes. The trial was randomised by undisclosed means and was unblinded. No patients withdrew and there were no crossover during the study period, thus earning this trial a score of two according to the Jadad criteria.

Kowey, et al conducted a single-centre retrospective cohort study of 112 AF-naïve patients who developed paroxysmal AF following coronary artery bypass grafting (CABG).[8] They compared rate control alone to rate control plus rhythm control with Class I or III antiarrhythmic agent (most commonly procainamide). They found no significant difference between the two groups in terms of length of hospital stay $(9.8 \pm 2.9 \vee 10.3 \pm 3.1, \mathrm{p}<0.05)$ or rates of AF at 6 weeks post-discharge $(1.32 \vee 2.78, \mathrm{p}>0.05)$. 
This cohort was somewhat representative of typical cardiac surgery cases with selection limited by the single-centre nature of the study and cohorts were drawn from the same community. The outcome was not present at study outset and ascertainment of exposure was based on secure data file review and record linkage (operation reports and clinical notes). Length (six weeks post-operative) and adequacy of followup were appropriate and complete respectively with all patients accounted for at six week follow-up aside from the seven in-hospital deaths that occurred during the study period. This trial scores seven stars according to the Newcastle-Ottawa scale, indicating a high-quality cohort study.

\section{Clinical bottom line}

AF is common following cardiac surgery with reported rates in the referenced studies $>30 \%$. Rate and rhythm control strategies are effective approaches with similar rates of adverse effects resulting in similar outcomes, importantly recurrence of arrhythmia and freedom from arrhythmia at follow-up. These findings are in line with findings from the much larger The Atrial Fibrillation Follow-up Investigation of Rhythm Management (AFFIRM) trial conducted among non-surgical patients with paroxysmal AF which show no clear advantage of one strategy over the other.[9] Choice of strategy in post-cardiac surgery patients does not greatly affect length of hospital stay but may be slightly shorter with rhythm control as time to conversion to sinus rhythm may be shorter in this group. Adverse events are similar between the groups as well. Trial design in this context clearly presents several major challenges in particular surrounding the issues of blinding and crossover. Choice of strategy should ultimately depend on patient characteristics, concurrent management issues, and physician expertise. 


\section{REFERENCES}

[1] Dunning J, Prendergast B, Mackway-Jones K. Towards evidence-based medicine in cardiothoracic surgery: best BETS. Interact CardioVasc Thorac Surg 2003;2:405-409.

[2] Berger VW, Alperson SY. A General Framework for the Evaluation of Clinical Trial Quality. Reviews on recent clinical trials. 2009;4:79-88.

[3] Wells GA, Shea B, O'Connell D, Peterson J, Welch V, Losos M, Tugwell P. The Newcastle-Ottawa Scale (NOS) for assessing the quality of nonrandomized studies in meta-analyses, http://www.ohri.ca/programs/clinical_epidemiology/oxford.asp.

[4] Gillinov AM, Bagiella E, Moskowitz AJ, Raiten JM, Groh MA, Bowdish ME, et al. Rate Control versus Rhythm Control for Atrial Fibrillation after Cardiac Surgery. N Engl J Med 2016;374:1911-1921.

[5] Lee JK, Klein GJ, Krahn AD, Yee R, Zarnke K, Simpson C, el al. Rate-control versus conversion strategy in postoperative atrial fibrillation: trial design and pilot study results. Card Electrophysiol Rev 2003;7:178-184.

[6] Cochrane A, Siddins M, Rosenfeldt R. A comparison of amiodarone and digoxin for treatment of supraventricular arrhythmias after cardiac surgery. Eur J Cardiothorac Surg 1994;8:194-198.

[7] Hjelms E. Procainamide conversion of acute atrial fibrillation after open-heart surgery compared with digoxin treatment. Scan J Thorac Cardiovasc Surg 1992;26:193-196.

[8] Kowey PR, Stebbins D, Igidbashian L, Goldman SM, Sutter FP, Rials SJ, et al. Clinical Outcome of Patients Who Develop PAF after CABG Surgery. J Pacing and Clin Electrophysiol 2001;24:191-193.

[9] Wyse DG, Waldo AL, DiMarco JP, Domanski MJ, Rosenberg Y, Schron EB, et al. A Comparison of Rate Control and Rhythm Control in Patients with Atrial Fibrillation. N Engl J Med 2002;347:1825-1833. 


\section{University Library}

\section{- M M I N E R VA A gateway to Melbourne's research publications}

Minerva Access is the Institutional Repository of The University of Melbourne

Author/s:

Conte, SM;Florisson, DS;De Bono, JA;Davies, RA;Newcomb, AE

Title:

Management of atrial fibrillation after cardiac surgery

Date:

2019-05-01

Citation:

Conte, S. M., Florisson, D. S., De Bono, J. A., Davies, R. A. \& Newcomb, A. E. (2019).

Management of atrial fibrillation after cardiac surgery. INTERNAL MEDICINE JOURNAL, 49 (5), pp.656-658. https://doi.org/10.1111/imj.14281.

Persistent Link:

http://hdl.handle.net/11343/285829 